\title{
Marginal structural model to evaluate the association between cumulative osteoporosis medication and infection using claims data
}

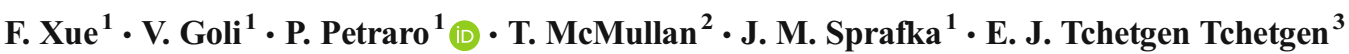

Received: 8 September 2016 / Accepted: 14 June 2017 / Published online: 6 July 2017

(C) The Author(s) 2017. This article is an open access publication

\begin{abstract}
Summary Due to the suboptimal persistence to osteoporosis (OP) treatment, factors triggering treatment discontinuation/switching may be causing time-varying confounding. BP treatment was associated with the risk of overall infection in opposite directions in the unweighted Cox model versus the weighted MSM. The discrepancy of effect estimates for overall infection in the MSM suggested there may be time-varying confounding.

Introduction Due to the suboptimal persistence to osteoporosis (OP) treatment, factors triggering treatment discontinuation/switching may be affected by prior treatment and confound the subsequent treatment effect, causing timevarying confounding.

Methods In a US insurance database, the association between joint treatment of bisphosphonates (BP) and other OP medication and the incidence of infections among postmenopausal women was assessed using a marginal structural model (MSM). Stabilized weights were esti-
\end{abstract}

Electronic supplementary material The online version of this article (doi:10.1007/s00198-017-4129-6) contains supplementary material, which is available to authorized users.

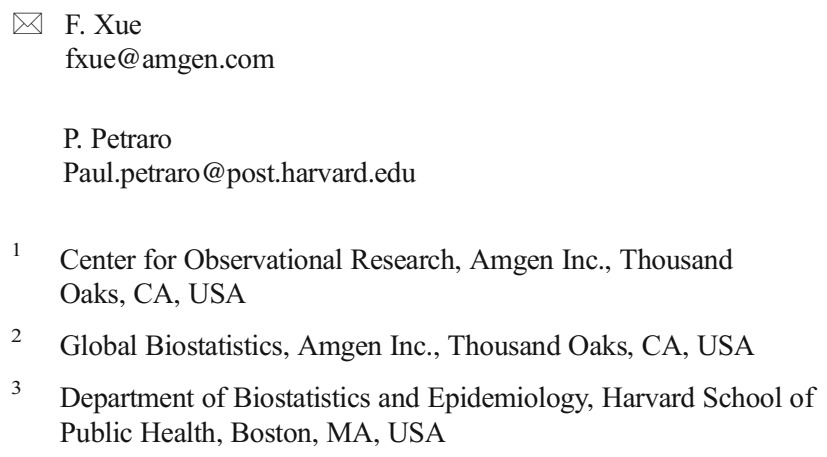

3 Department of Biostatistics and Epidemiology, Harvard School of Public Health, Boston, MA, USA

mated by modeling treatment and censoring processes conditioning on past treatment, and baseline and timevarying covariates.

Results BP treatment was associated with the risk of overall infection in opposite directions in the unweighted Cox model \{incidence rate ratio [IRR] [95\% confidence interval $(\mathrm{CI})]=1.15[1.14-1.17]\}$ versus the weighted MSM [IRR $(95 \% \mathrm{CI})=0.79(0.77-0.81)]$, but was consistently associated with a lower risk of serious infection in both the unweighted Cox model $[\mathrm{IRR}(95 \% \mathrm{CI}]=0.79(0.78-0.81))$ and the weighted MSM [IRR $(95 \%$ CI $)=0.71(0.68-0.75)]$. Similar results were found when current and past treatments were simultaneously assessed.

Conclusions The discrepancy of effect estimates for overall but not serious infection comparing unweighted models and MSM suggested analyses of composite outcomes with a wide range of disease severity may be more susceptible to timevarying confounding.

Keywords Claims $\cdot$ Infection $\cdot$ MSM $\cdot$ Osteoporosis

\begin{tabular}{ll}
\multicolumn{2}{l}{ Abbreviations } \\
BP & Bisphosphonates \\
CI & Confidence interval \\
ICD-9-CM & $\begin{array}{l}\text { International Classification of Disease, } \\
\text { Ninth Revision, Clinical Modification }\end{array}$ \\
IRR & $\begin{array}{l}\text { Incidence rate ratio } \\
\text { MSM }\end{array}$ \\
ONJ & Osteonal structural model \\
OP & Osteoporosis of the jaw \\
PH & Proportional hazards \\
PMO & Postmenopausal osteoporosis
\end{tabular}




\section{Introduction}

Bisphosphonates (BP) are the most commonly prescribed medications to treat osteoporosis (OP). They are potent anti-resorptive agents that maintain or increase bone strength by slowing or preventing the dissolving of bone [1]. BP treatment has been associated with an increased risk of osteonecrosis of the jaw (ONJ) $[2,3]$. While the underlying mechanism for antiresorptive therapy to cause ONJ is unknown, infection was suggested to play a pivotal role in the pathogenesis of ONJ and persistent infection may prevent bone and soft tissue lesion from healing [4]. A histological analysis of ONJ cases treated with BP also suggested that the development of ONJ appears to be an inflammationassociated process [5]. It remains unclear whether BP treatment affects the risk of infection. Despite a few case reports, there is no population-based study that systematically evaluated the potential effect of BP treatment on the risk of infection.

One challenge in assessing treatment effects of BP is the poor persistence with these medications as a result of inconvenient dosing regimens, drug side effects or other comorbid conditions, especially in women with postmenopausal osteoporosis (PMO) [6]. Therefore, the likelihood of time-varying confounding is increased when comparing the long-term treatment effect of BP versus other OP medications since earlier OP medications may affect occurrence of the study outcome as well as subsequent OP treatments. For instance, BP and other OP medications may have different potency in reducing the risk of fragility fracture, and fracture is known to be a risk factor for infection. Time-varying confounding by fragility fracture for the association between sequential OP treatments and the risk of infection is depicted in the causal directed acyclic graph in Fig. 1. Because fragility fracture is both a mediating factor for the potential effect of the OP treatment 1 on the risk of infection, and a time-dependent confounder for the association between the OP treatment 2 and the

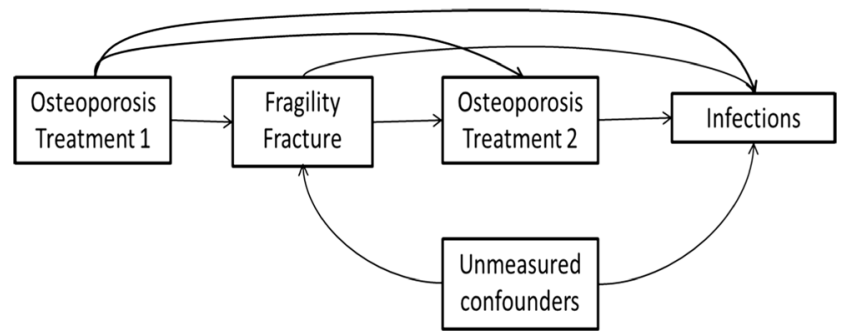

Fig. 1 Directed acyclic graph demonstrating time-varying confounding by fragility fracture of the association between sequential OP treatments and risk of infections risk of infection, either adjusting or not adjusting for fragility fracture using standard confounding adjustment methods (e.g., covariate-adjustment regression model or stratification) will generate biased effect estimates [7, 8]. In the presence of unmeasured confounders on the association between fragility fracture and risk of serious infections that cannot be ruled out with certainty, such as cigarette smoking or malnutrition, fragility fracture can be a collider (i.e., outcome of two or more variables) $[9,10]$ and thus conditioning on it in a regression model would open a back-door path, producing additional bias [11]. Instead the influence of such timevarying confounder(s) on the overall sequential treatment effects can be accounted for by inverse probability of treatment weighted (IPTW) estimation of the parameters in a marginal structural model (MSM) for the joint effect of treatment history on the incidence of infection $[7,12]$. In the example described above, conceptually, a pseudo-population can be created through weighting each subject in the study population by the inverse probability of receiving the OP treatment history they indeed received, conditional on their history of timefixed and time-varying confounders (e.g., fragility fracture). In the resulting pseudo-population, fragility fracture no longer affects the probability of the subsequent OP treatment, and therefore is no longer a time-varying confounder; however, the causal association between treatment history and infection risk remains as in the original population. Thus, an analysis that does not adjust for time-varying confounders in the pseudopopulation will generate an unbiased effect estimate of the joint causal effect of sequential OP treatment, assuming no other unmeasured confounders are present. A similar approach can also be used to handle censoring due to loss to follow-up by conceptualizing censoring as another time-varying treatment $[7,13]$. Numerous other time-varying variables affected by earlier OP treatment may directly or indirectly influence patients' future choice of OP treatment and causing time-varying confounding. The potential channeling bias that BP treatment is more likely to be prescribed to heathier patients who are perceived to be at lower risk of known related adverse events may also contribute to time-varying confounding as prior BP versus other OP treatment may have different potency in improving the bone and overall health of a patient.

In the current study, we evaluated the association between treatment history (BP and other OP medication) and the incidence rate of infections using marginal structural Cox proportional hazards (PH) models adjusting for fragility fracture and other time-varying covariates. As there is no anticipation of treatment effect with regard to specific anatomic site or pathogen 
type, overall infection identified by any infection diagnosis from a large insurance claims database was assessed. Serious infections leading to hospitalization, ER visit, or administration of parenteral anti-infective medication use were also evaluated for better specificity of outcome assessment. To assess the impact of timevarying confounding in this study, the same association was also estimated through regular unweighted multivariate-adjusted Cox PH models.

\section{Methods}

\section{Study population}

The analyses were conducted in the commercial claims and Medicare Coordination of Benefits databases from MarketScan, including data from 01 January 2004 through 30 June 2011. The MarketScan database captures person-specific clinical utilization, expenditures, and enrollment across inpatient, outpatient, prescription drug, and carve-out services from a selection of large employers, health plans, and government and public organizations.

Women with PMO were included in the study based on the following inclusion criteria during the study period: postmenopausal age ( $\geq 55$ years); had $\geq 12$ months of continuous enrollment in the health plan; and had a diagnosis of OP or osteoporotic fracture or treatment with an OP medication. The PMO index date of an eligible woman was defined by the earliest date when she satisfied all of the above inclusion criteria during the study period. The 12-month period before the PMO index date was defined as the PMO baseline period, during which patient characteristics including exposure to OP medications and timefixed covariates were assessed (Fig. 2). Women with a diagnosis for Paget's disease or a diagnosis or treatment related to malignancy (excluding non-melanoma skin cancer) during the PMO baseline period were excluded.

\section{Exposure assessment}

OP treatment including BP and other OP medications were assessed through drug codes or procedure codes. BP were restricted to those indicated for the treatment of PMO, i.e., IV BP including zoledronic acid infusion (Reclast) and ibandronate injection, and oral BP including alendronate sodium, alendronate sodium/vitamin D tablet (Fosamax plus D), ibandronate sodium, risedronate and risedronate sodium/calcium carbonate. Other OP medications included teriparatide injection, raloxifene tablet, and calcitonin.
The lack of regularly scheduled visits in studies based on administrative claims data poses a challenge that standard epidemiologic cohort studies do not face. To address this issue, an unstructured "visit process" was used to assess time-varying treatment with OP medications (BP or other) during the follow-up for all patients. From the PMO index date until the end of follow-up, each OP medication received was flagged. In our study design, a visit was defined by the initiation of an OP treatment, switching to the other treatment or reaching 6-month following switching to no treatment (see Fig. 2 footnote for definition of switching), diagnosis of fragility fracture, diagnosis of study outcome (infection) or end of follow-up (see the "Methods" section on follow-up for details). These specific events are likely to indicate or influence the decision on patients' choice of OP medication and thus updating covariates prior to these events provides important assessment of potential time-varying confounding variables. Associated with each visit is a look-back period defined as the 6month prior to the visit (including the visit date). The look-back period was used to assess exposure to OP medications, as well as time-varying covariates for each visit. PMO baseline period can be the earliest look-back period for a patient.

Figure 2 displays visits and look-back periods for a hypothetical patient. In this example, the patient received a BP at visit 1 (PMO index date) and other OP medication at visit 2 (month 2), then switched back to $\mathrm{BP}$ at visit 3 (month 5). Assuming that the days supplied for the second BP is 30 days and with a buffer of 60 days, the patient is deemed to have switched to no treatment at month 8 .

The exposure to OP medications was classified using the following two approaches to account for treatment history of OP medications:

1. Categorical classification based on the treatment received in the current look-back period (BP including both $\mathrm{BP}$ and other OP medication, other OP medication only and no OP medication) as well as treatment received in the current period and any of the past look-back periods (current BP treatment only, current and past BP treatment, past BP treatment only, current other OP medications only, current other OP medications and past BP treatment, and no OP medication).

2. Quintiles of cumulative dosage of BP treatment received during all previous look-back periods calculated through standardizing the received dose of various BP to alendronate-equivalent daily dose (Appendix A). The midpoint of each quintile was also used as a categorical variable to test the significance of trend. 


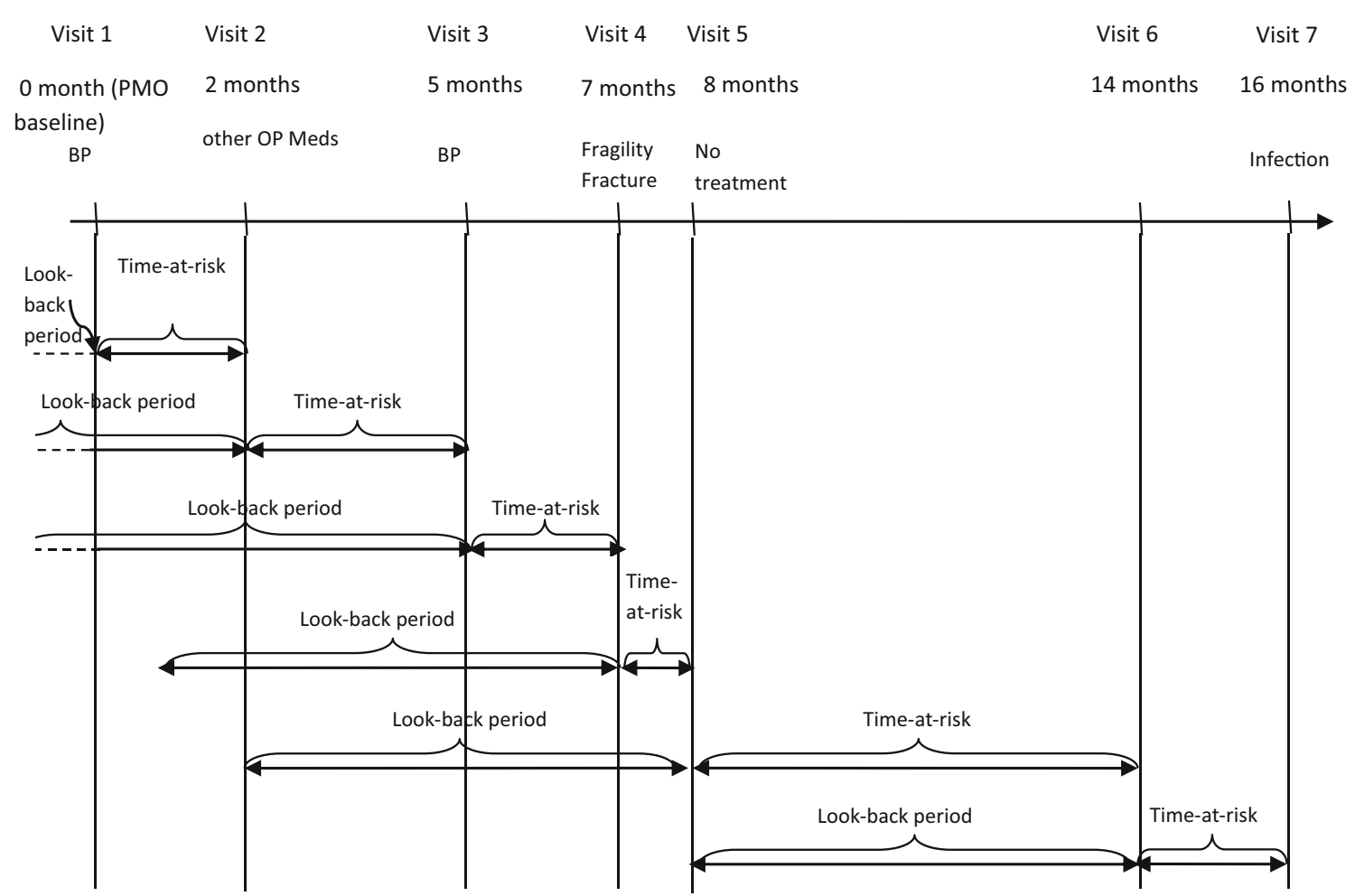

Fig. 2 An example of how to flag "visit" for a patient by switching of treatments, occurrence of a fragility fracture, and experience of the outcome of interest (infection). Schematic view of the 6-month look-back period (from the current visit) and the time-at-risk for the outcome (i.e., the day after the current visit up to and including the day of the next visit). Treatment switching status was defined as follows: (1) If a patient continuously received multiple treatment of the same class of drugs (BP or other OP medications) with a gap between two doses of less than the number of days supplied of the previous dose plus 60 days, the patient

\section{Outcome assessment}

Diagnosis of overall infection and serious infection was identified through relevant International Classification of Disease, Ninth Revision, Clinical Modification (ICD-9-CM), treatment codes, and procedure codes throughout the eligible follow-up period for each patient. Overall infections included a wide range of infection types without restriction by specific pathogen or anatomic site, and serious infections included the same infection types but required the infection was severe enough to lead to hospitalization, ER visit, or administration of parenteral anti-infective medication use. The requirement of additional claims-identifiable medical procedures or drug treatment for the assessment of serious infection is intended to enhance the specificity and reduce false-positive of study outcome. The outcome status was updated and time at risk was calculated as the time from current visit to next visit (Fig. 2).

\section{Covariate assessment}

A variety of time-fixed and time-varying covariates were assessed, including demographic characteristics, was deemed as staying on the same treatment. (2) If a patient received OP medication other than BP before the end of days supplied of the previous $\mathrm{BP}$ treatment plus 60 days or vice versa, the patient was deemed as having switched to the other treatment. (3) If a patient did not receive the same or different OP medication after the end of days supplied plus 60 days of the previous treatment, the patient was deemed as having switched to no treatment. (4) If a patient received BP or other OP medication after having switched to no treatment, the patient was deemed as having switched to the $\mathrm{BP}$ or other OP medication, respectively

comorbidities and concomitant medications which are potential risk factors for infection as well as healthcare utilization variables (Appendix B). The time-fixed covariates were evaluated only once during the PMO baseline period and time-varying covariates were updated during each look-back period. If the timevarying covariate was a chronic disease (e.g., diabetes), once the diagnosis was identified, it was carried forward throughout the remaining follow-up period.

\section{Follow-up}

All women with PMO were followed from the PMO index date until the first of the following: disenrollment from the data system, diagnosis or treatment related to malignancy (excluding non-melanoma skin cancer), diagnosis of Paget's disease, or end of the study period (30 June 2011). The follow-up for the occurrence of overall infection and serious infection was independently conducted, so that the occurrence of one outcome did not censor the follow-up for the other outcomes. 


\section{Statistical analysis}

The association between OP treatment and the incidence of overall infection and serious infection was evaluated through MSM based on IPTW. The stabilized weight for MSM was calculated in the steps described in the Appendix C [14].

The incidence rate ratio (IRR) of overall infection and serious infection comparing the OP treatment groups was estimated using a Cox regression MSM model. Time-fixed covariates and time since start of follow-up (modeled as natural cubic splines) were also included in the model as timedependent intercepts. Robust standard errors were used to construct confidence interval (CI) for MSM parameter estimates. The association between treatment with OP medications and the incidence of overall infection and serious infection was also estimated through standard Cox regression models without weighting by SWi(t) treatment or SWi $(\mathrm{t})_{\text {censoring. Unadjusted and two sets of multivariate- }}$ adjusted analyses were performed. In the first set of analyses, only time-fixed covariates were adjusted for, while in the second set of analyses, both time-fixed and time-varying covariates were adjusted for.

\section{Results}

During the study period, a total of 469,432 women with PMO were identified from the MarketScan insurance claims database and contributed 1,050,567 person-years of follow-up. Among them, 464,728, 67,860 and 517,979 person-years received $\mathrm{BP}$, other OP medications, and no OP medication, respectively, during the look-back period before each visit (Table 1). Women who received a BP or other OP treatment were younger and had a lower prevalence of several comorbidities such as diabetes, end stage renal disease, chronic obstructive pulmonary disease, chronic lung disease, serious infection, fragility fracture and other fracture than women who received no OP treatment. When cohorts were classified based on BP and other OP medications received in the current period and any of the past look-back periods, the same pattern was observed that treated cohorts were younger, and less likely to have comorbidities than the untreated cohort (Table 1).

The polytomous logistic regression models used to estimate treatment weights are displayed in Appendix D. Since including extreme weights would cause instability of weighted estimates of the MSM, the stabilized weights were truncated at a threshold percentile level (i.e., 0.5 and $99.5 \%$ in primary analysis and 1 and $99 \%$ in sensitivity analysis), so that patients with stabilized weight more extreme than the threshold level were set to the threshold level (Table 2). In additional analyses, treatment and censoring models derived from the most recent four previous visits only were used to construct the stabilized weight to limit the influence of extreme weights and explore whether recent diagnoses and treatment are more influential on OP treatment and censoring.

In the analysis for overall infection, treatment with BP and other OP medications during the 6-month look-back period was associated with an increased risk in the unweighted Cox regression model [IRR $(95 \% \mathrm{CI})=1.15(1.14-1.17)$ and 1.25 (1.23-1.27), respectively] relative to the untreated cohort (Table 3). The association became weaker for both treatments after adjustment for time-fixed variables only or both timefixed and time-varying variables. The direction of the association between treatment and the risk of overall infection was reversed in the MSM for BP [IRR $(95 \% \mathrm{CI})=0.79(0.77-$ $0.81)$ ] and other OP medications [IRR $(95 \% \mathrm{CI})=0.86(0.82-$ $0.90)]$. The results on combinations of cumulative OP treatment followed the same pattern that the categories with current and/or past BP treatment were associated with a lower risk of overall infection in the MSM while the association between current BP treatment and overall infection was reversed in the unweighted Cox regression models. Furthermore, higher dose quintiles of cumulative BP treatment was found to be associated with a lower risk of overall infection [IRR $(95 \% \mathrm{CI})=0.98(0.95-1.01), 0.96(0.93-0.98)$, $0.91(0.88-0.93), 0.81(0.79-0.84)$ for $300-859 \mathrm{mg}, 860-$ $1799 \mathrm{mg}, 1800-4039 \mathrm{mg}$ and $\geq 4040 \mathrm{mg}$ relative to $1-$ $299 \mathrm{mg}$, respectively, in MSM] ( $P$ for trend $<0.001$ ).

In the analysis for serious infection, current and past BP treatment was consistently associated with a lower risk relative to those untreated in unweighted Cox regression models as well as MSM (Table 4). A dose-response trend was also observed in the association between cumulative dose of BP treatment and the lower risk of serious infection in the unweighted analysis $(P$ for trend $<0.001)$ but the association was not present in the MSM ( $P$ for trend $=0.63$ ).

Sensitivity analysis of MSM by truncating stabilized combined weight at 1 and 99 percentile or based on stabilized weights derived from the most recent four visits produced similar results to the primary analysis.

\section{Discussion}

In this retrospective cohort study among women with $\mathrm{PMO}$, patients treated with BP were found to have a lower risk of serious infection leading to hospitalization or ER visit in both unweighted multivariate-adjusted models and the MSM weighted analysis. The same association between treatment with BPs and a lower risk of overall infection was observed with the MSM but not unweighted models.

The descriptive analysis of baseline characteristics suggested patients treated with BP and other OP medications were 


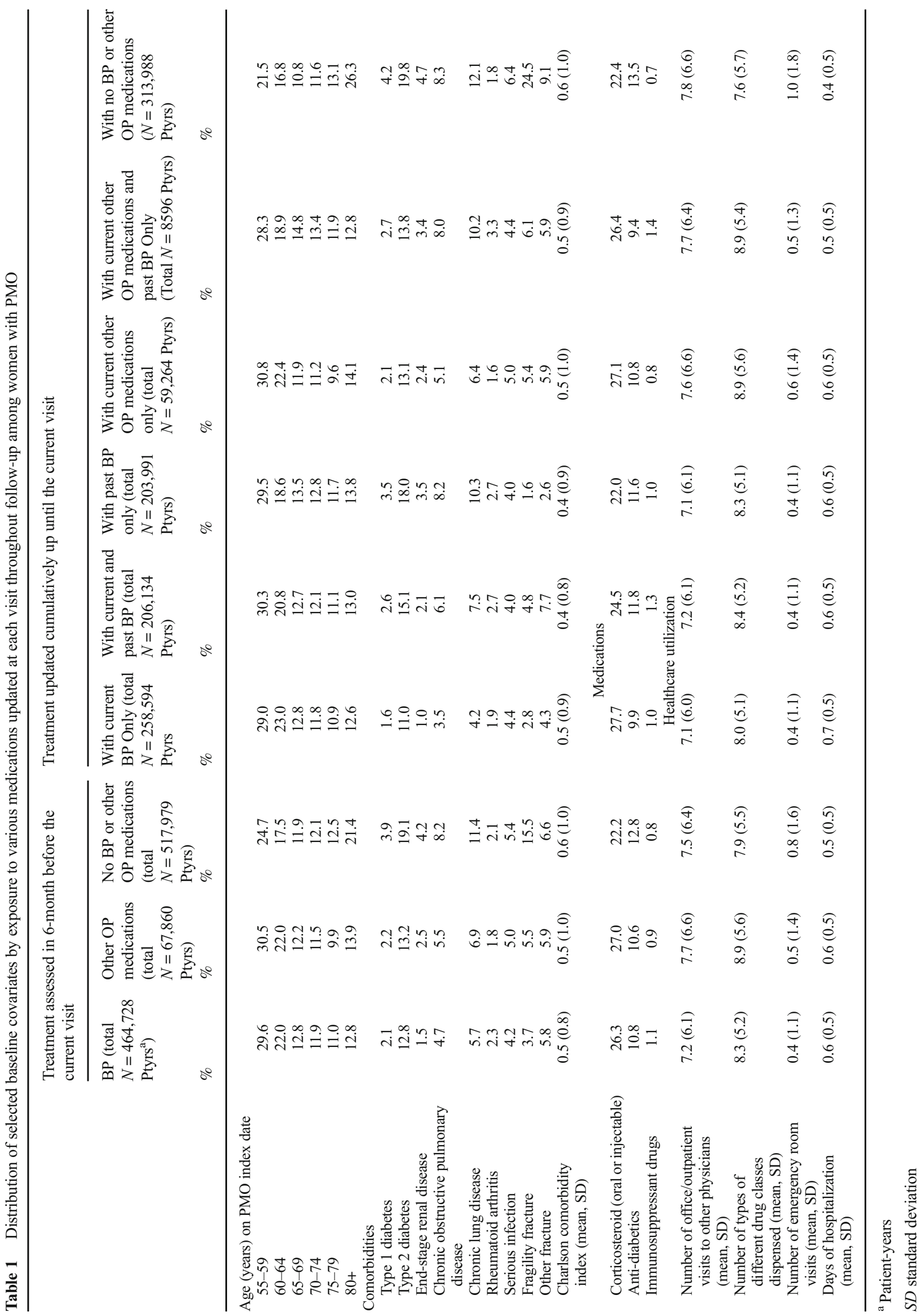


Table 2 Distribution of logstabilized combined weights at each visit for the analysis of serious infection and overall infection with and without truncation at the $0.5 \%$ and $99.5 \%$ percentile

\begin{tabular}{|c|c|c|c|c|c|c|c|}
\hline Outcome & & Minimum & $\begin{array}{l}25 \% \\
\text { percentile }\end{array}$ & Mean & Median & $\begin{array}{l}75 \% \\
\text { percentile }\end{array}$ & Maximum \\
\hline \multirow{2}{*}{$\begin{array}{l}\text { Overall } \\
\text { infection }\end{array}$} & Original & 0 & 0.26 & 1.79 & 0.59 & 0.94 & $201,071.49$ \\
\hline & Truncated & 0 & 0.26 & 0.89 & 0.59 & 0.94 & 19.74 \\
\hline \multirow{2}{*}{$\begin{array}{l}\text { Serious } \\
\text { infection }\end{array}$} & Original & 0 & 0.19 & 1.25 & 0.52 & 0.91 & $32,770.96$ \\
\hline & Truncated & 0 & 0.19 & 0.85 & 0.52 & 0.91 & 18.71 \\
\hline
\end{tabular}

younger and had a lower prevalence of selected risk factors of infection. The identification of serious infections leading to hospitalization, ER visit, or administration of parenteral antiinfective medication use is less susceptible to false-positive than overall infections. Nonetheless, overall infections, as a more sensitive outcome, was included in this study to evaluate the consistency of the association of OP treatment with the risk of a broader range of infectious conditions including less severe and local infections. Direct adjustment for potential risk factors of infection in the regression models generated opposite results on the association of BP with overall infection versus serious infection. These results were not anticipated given that serious infections are the more severe types of overall infection and they are expected to share a similar etiology and risk factors. On the other hand, when these risk factors were used in the weights for IPTW estimation of a MSM, treatment with BP was consistently associated with a decreased risk of overall infection and serious infection. The reduced risk of infection associated with BP was further supported by the dose-response observed for both serious infection and overall infection. These

Table 3 IRR and 95\% CI of overall infection comparing OP medication cohorts derived from MSM and regular Cox regression model

\begin{tabular}{|c|c|c|c|c|c|c|c|c|c|c|}
\hline \multirow[t]{2}{*}{ OP treatment } & \multirow[t]{2}{*}{$\begin{array}{l}\text { Number of } \\
\text { patient-years }\end{array}$} & \multirow[t]{2}{*}{$\begin{array}{l}\text { Number of } \\
\text { cases }\end{array}$} & \multicolumn{2}{|c|}{$\begin{array}{l}\text { Unadjusted Cox } \\
\text { PH regression } \\
\text { model }\end{array}$} & \multicolumn{2}{|c|}{$\begin{array}{l}\text { Multivariate Cox } \\
\text { PH regression } \\
\text { model I* }\end{array}$} & \multicolumn{2}{|c|}{$\begin{array}{l}\text { Multivariate Cox } \\
\text { PH regression } \\
\text { model } \mathrm{II}^{\dagger}\end{array}$} & \multicolumn{2}{|c|}{$\mathrm{MSM}^{*}$} \\
\hline & & & IRR & $95 \% \mathrm{CI}$ & IRR & $95 \% \mathrm{CI}$ & IRR & $95 \% \mathrm{CI}$ & IRR & $95 \% \mathrm{CI}$ \\
\hline \multicolumn{11}{|c|}{ Treatment assessed in 6 months before the current visit } \\
\hline Without treatment & 330,429 & 78,634 & 1 & - & 1 & - & 1 & - & 1 & - \\
\hline BP only & 335,976 & 82,963 & 1.15 & $(1.14-1.17)$ & 1.11 & $(1.10-1.13)$ & 1.07 & $(1.05-1.08)$ & 0.79 & $(0.77-0.81)$ \\
\hline Other OP medication only & 47,433 & 12,882 & 1.25 & $(1.23-1.27)$ & 1.17 & $(1.14-1.19)$ & 1.11 & $(1.08-1.13)$ & 0.86 & $(0.82-0.90)$ \\
\hline \multicolumn{11}{|c|}{ Treatment updated cumulatively up until the current visit } \\
\hline Without treatment & 221,126 & 58,148 & 1 & - & 1 & - & 1 & - & 1 & - \\
\hline With current BP only & 211,083 & 54,117 & 1.15 & $(1.13-1.17)$ & 1.11 & $(1.09-1.12)$ & 1.02 & $(1.01-1.04)$ & 0.86 & $(0.84-0.88)$ \\
\hline With current and past BP only & 124,893 & 28,846 & 0.93 & $(0.97-0.94)$ & 0.95 & $(0.93-0.96)$ & 1.04 & $(1.03-1.06)$ & 0.75 & $(0.72-0.78)$ \\
\hline With Past BP only & 109,302 & 20,486 & 0.71 & $(0.70-0.73)$ & 0.77 & $(0.76-0.79)$ & 0.88 & $(0.86-0.89)$ & 0.75 & $(0.72-0.78)$ \\
\hline With current other OP medication only & 43,134 & 11,968 & 1.18 & $(1.15-1.20)$ & 1.11 & $(1.09-1.13)$ & 1.09 & $(1.06-1.11)$ & 0.89 & $(0.85-0.93)$ \\
\hline $\begin{array}{l}\text { With current other OP medication } \\
\text { and past BP Only }\end{array}$ & 4299 & 914 & 0.88 & $(0.82-0.94)$ & 0.91 & $(0.86-0.98)$ & 1.02 & $(0.95-1.09)$ & 0.79 & $(0.66-0.95)$ \\
\hline \multicolumn{11}{|c|}{ Cumulative BP dose — standardized to alendronate } \\
\hline $1-299 \mathrm{mg}$ & 41,591 & 10,340 & 1 & - & 1 & - & 1 & - & 1 & - \\
\hline $300-859 \mathrm{mg}$ & 55,002 & 12,875 & 0.94 & $(0.92-0.97)$ & 0.94 & $(0.92-0.97)$ & 0.96 & $(0.94-0.99)$ & 0.98 & $(0.95-1.01)$ \\
\hline $860-1799 \mathrm{mg}$ & 49,490 & 10,564 & 0.86 & $(0.84-0.88)$ & 0.88 & $(0.86-0.90)$ & 0.91 & $(0.89-0.94)$ & 0.96 & $(0.93-0.98)$ \\
\hline $1800-4039 \mathrm{mg}$ & 47,500 & 9175 & 0.78 & $(0.76-0.80)$ & 0.83 & $(0.80-0.85)$ & 0.86 & $(0.84-0.88)$ & 0.91 & $(0.88-0.93)$ \\
\hline$\geq 4040 \mathrm{mg}$ & 45,188 & 7381 & 0.66 & $(0.64-0.68)$ & 0.75 & $(0.72-0.77)$ & 0.78 & $(0.76-0.81)$ & 0.81 & $(0.79-0.84)$ \\
\hline$P$ for trend & 238,771 & 50,335 & & $<0.001$ & & $<0.001$ & & $<0.001$ & & $<0.001$ \\
\hline
\end{tabular}

*Adjusted for time-fixed variables including geographic region, length of enrollment in the data system, calendar year, number of office/outpatient visits, number of type of different drugs dispensed, number of emergency room visits, and days of hospitalization.

${ }^{\dagger}$ Adjusted for above mentioned time-fixed variables and time-varying variables including age, diabetes (type I and type II), end stage renal disease, HIV/ AIDS, liver cirrhosis, cystic fibrosis, chronic obstructive pulmonary disease, lupus, multiple sclerosis, rheumatoid arthritis, ankylosing spondylitis, Crohn's disease, inflammatory bowel disease, psoriasis, rheumatic fever, overweight/obesity, serious infection, serious neutropenia, decubitus ulcer, fragility fracture, Charlson comorbidity index, bisphosphonate treatment, corticosteroid (oral or injectable), anti-diabetics and immunosuppressant drugs, and malnutrition.

* Adjusted for abovementioned time-fixed variables with stabilized weight truncated at 0.5 th and 99.5 th percentile. 
Table 4 IRR and 95\% CI of serious infection comparing OP medication cohorts derived from MSM and regular Cox regression model

\begin{tabular}{|c|c|c|c|c|c|c|c|c|c|c|}
\hline \multirow[t]{2}{*}{ OP treatment } & \multirow[t]{2}{*}{$\begin{array}{l}\text { Number of } \\
\text { patient-years }\end{array}$} & \multirow[t]{2}{*}{$\begin{array}{l}\text { Number of } \\
\text { cases }\end{array}$} & \multicolumn{2}{|c|}{$\begin{array}{l}\text { Unadjusted Cox } \\
\text { PH regression } \\
\text { model }\end{array}$} & \multicolumn{2}{|c|}{$\begin{array}{l}\text { Multivariate Cox } \\
\text { PH regression } \\
\text { model I* }\end{array}$} & \multicolumn{2}{|c|}{$\begin{array}{l}\text { Multivariate Cox } \\
\text { PH regression } \\
\text { model } \mathrm{II}^{\dagger}\end{array}$} & \multicolumn{2}{|c|}{$\mathrm{MSM}^{\ddagger}$} \\
\hline & & & IRR & $95 \% \mathrm{CI}$ & IRR & $95 \% \mathrm{CI}$ & IRR & $95 \% \mathrm{CI}$ & IRR & $95 \% \mathrm{CI}$ \\
\hline \multicolumn{11}{|c|}{ Treatment assessed in 6-month before the current visit } \\
\hline Without treatment & 448,182 & 37,316 & 1 & - & 1 & - & 1 & - & 1 & - \\
\hline BP only & 430,433 & 25,191 & 0.79 & $(0.78-0.81)$ & 0.78 & $(0.77-0.79)$ & 0.91 & $(0.89-0.93)$ & 0.71 & $(0.68-0.75)$ \\
\hline Other OP medication only & 62,122 & 4263 & 0.92 & $(0.89-0.95)$ & 0.86 & $(0.83-0.89)$ & 0.97 & $(0.93-1.01)$ & 0.77 & $(0.71-0.83)$ \\
\hline \multicolumn{11}{|c|}{ Treatment updated cumulatively up until the current visit } \\
\hline Without treatment & 276,254 & 26,945 & 1 & - & 1 & - & 1 & - & 1 & - \\
\hline With current BP only & 253,210 & 14,257 & 0.68 & $(0.67-0.70)$ & 0.68 & $(0.66-0.70)$ & 0.84 & $(0.82-0.86)$ & 0.65 & $(0.62-0.68)$ \\
\hline With current and past BP Only & 177,223 & 10,934 & 0.67 & $(0.66-0.69)$ & 0.68 & $(0.67-0.70)$ & 0.90 & $(0.88-0.93)$ & 0.96 & $(0.90-1.01)$ \\
\hline With past BP only & 171,927 & 10,371 & 0.62 & $(0.61-0.63)$ & 0.66 & $(0.65-0.68)$ & 0.86 & $(0.84-0.88)$ & 0.89 & $(0.84-0.94)$ \\
\hline With current other OP medication only & 54,945 & 3829 & 0.80 & $(0.77-0.83)$ & 0.76 & $(0.73-0.78)$ & 0.94 & $(0.91-0.97)$ & 0.86 & $(0.79-0.93)$ \\
\hline $\begin{array}{l}\text { With current other OP medication } \\
\text { and past BP only }\end{array}$ & 7178 & 434 & 0.67 & $(0.61-0.74)$ & 0.69 & $(0.62-0.75)$ & 0.87 & $(0.79-0.95)$ & 1.09 & $(0.88-1.36)$ \\
\hline \multicolumn{11}{|c|}{ Cumulative BP dose — standardized to alendronate } \\
\hline $1-430 \mathrm{mg}$ & 73,520 & 5233 & 1 & - & 1 & - & 1 & - & 1 & - \\
\hline $430-900 \mathrm{mg}$ & 69,134 & 4370 & 0.89 & $(0.85-0.93)$ & 0.91 & $(0.87-0.94)$ & 0.92 & $(0.88-0.96)$ & 0.97 & $(0.93-1.01)$ \\
\hline $900-2240 \mathrm{mg}$ & 76,171 & 4746 & 0.88 & $(0.85-0.92)$ & 0.90 & $(0.86-0.93)$ & 0.91 & $(0.88-0.95)$ & 0.99 & $(0.95-1.03)$ \\
\hline $2240-4780 \mathrm{mg}$ & 73,769 & 4103 & 0.79 & $(0.76-0.82)$ & 0.83 & $(0.80-0.87)$ & 0.85 & $(0.82-0.89)$ & 1.01 & $(0.97-1.05)$ \\
\hline$\geq 4780 \mathrm{mg}$ & 64,072 & 3311 & 0.74 & $(0.70-0.77)$ & 0.81 & $(0.78-0.85)$ & 0.81 & $(0.78-0.85)$ & 0.98 & $(0.94-1.03)$ \\
\hline $\mathrm{P}$ for Trend & 356,666 & 21,763 & & $<0.001$ & & $<0.001$ & & $<0.001$ & & 0.63 \\
\hline
\end{tabular}

*Adjusted for time-fixed variables including geographic region, length of enrollment in the data system, calendar year, number of office/outpatient visits, number of type of different drugs dispensed, number of emergency room visits, and days of hospitalization.

${ }^{\dagger}$ Adjusted for abovementioned time-fixed variables and time-varying variables including age, diabetes (type I and type II), end stage renal disease, HIV/ AIDS, liver cirrhosis, cystic fibrosis, chronic obstructive pulmonary disease, lupus, multiple sclerosis, rheumatoid arthritis, ankylosing spondylitis, Crohn's disease, inflammatory bowel disease, psoriasis, rheumatic fever, overweight/obesity, serious infection, serious neutropenia, decubitus ulcer, fragility fracture, Charlson comorbidity index, bisphosphonate treatment, corticosteroid (oral or injectable), anti-diabetics and immunosuppressant drugs, and malnutrition.

$\star$ Adjusted for abovementioned time-fixed variables with stabilized weight truncated at 0.5 th and 99.5 th percentile.

results suggest that time-varying confounding is only present in the comparison of the infection risk between treated and untreated OP patients. Furthermore, given the wide range of conditions included in the assessment of overall infection, specific time-varying confounders may only be present for less severe infections and thus only MSM rather than regular multivariateadjusted model can generate a valid effect estimate.

Though the underlying mechanism for the reduced risk of infection associated with BP treatment is unknown, potential influence of BP on the immune system has been documented based on animal studies and in vitro studies. Administration of clinically relevant doses of BP in mice increases antibody response to live and inactive viruses, proteins, haptens, and existing commercial vaccine formulations. It was found that BP target $\mathrm{B}$ cells and enhance $\mathrm{B}$ cell expansion and antibody production upon encounter, suggesting BP are an additional class of adjuvants that boost humoral immune responses [15]. In addition, BP may exert several effects on the immune system. In particular, pamidronate stimulates the production of a $\mathrm{T}$ cell subset that specifically inhibits bone resorption and may activate the production of antigenic receptor $\mathrm{T}$ cells, leading to the release of cytokines $[16,17]$. Other studies also reported other effects of BP in increasing the production of interleukin-1, interleukin-6, and Creactive proteins [16-19]. Future human studies including population-based studies are warranted to confirm whether these changes in the immune system induced by BP treatment convey a protective effect against infection.

Several additional limitations of the current study should be noted when interpreting the results. First, claims databases have inherent limitations because the claims are collected for the purpose of payment rather than research. For instance, the presence of a diagnosis code on a medical claim may not represent the true presence of a disease, as the diagnosis code may be incorrectly coded or included as rule-out criteria rather than actual disease. Secondly, the assessment of cumulative dose of BP was based on treatment received during all previous look-back periods, and 
thus the length of available look-back period partially determines the total cumulative dose. Therefore, the assessment of doseresponse relationship with BP treatment may be biased towards the null given the likely non-differential misclassification of the cumulative dose. Thirdly, the unstructured "visit process" used in the study does not differentiate the temporal sequence between the time-varying confounders and time-varying exposure during the look-back period. Therefore, for some patients time-varying confounders may be affected by received OP treatment. Fourthly, because time at risk is defined as the interval between two adjacent visits, it may not reflect the actual etiologically relevant time window. Finally, the estimated effect in this study was based on the observed visit process and therefore may not be generalized to a population with different visit process.

A reduced risk of serious and overall infection associated with BP treatment in women with PMO was identified in this study using MSM to adjust for time-varying confounders. The discrepancy of effect estimates for overall infection but not serious infection comparing unweighted multivariateadjusted models and MSM suggested the analysis of composite outcomes with a wide range of disease severity may be more susceptible to time-varying confounding. Further studies need to confirm these findings and investigate potential underlying mechanisms.

Acknowledgements This work was supported by Amgen Inc.

\section{Compliance with ethical standards}

Conflicts of interest Fei Xue, Vamshidar Goli, Paul Petraro, Trevor McMullan, and J. Michael Sprafka were employees of Amgen at the time of this manuscript preparation. Fei Xue and J. Michael Sprafka held Amgen stocks/stock options when the manuscript was prepared. Eric Tchetgen Tchetgen declares that he has no conflict of interest.

Open Access This article is distributed under the terms of the Creative Commons Attribution-NonCommercial 4.0 International License (http:// creativecommons.org/licenses/by-nc/4.0/), which permits any noncommercial use, distribution, and reproduction in any medium, provided you give appropriate credit to the original author(s) and the source, provide a link to the Creative Commons license, and indicate if changes were made.

\section{References}

1. DiPiro JT, Talbert RL, Yee GC, Matzke GR, Wells BG, Posey LM (2005) Pharmacotherapy: a pathophysiologic approach. McGrawHill, New York
2. Fellows JL, Rindal DB, Barasch A et al (2011) ONJ in two dental practice-based research network regions. J Dent Res 90:433-438

3. Chang JI, Hazboun RC, Chang TI (2014) Incongruities in the AAOMS Position Paper: Medication-Related Osteonecrosis of the Jaw-2014 Update. J Oral Maxillofac Surg 72(12):2381

4. Reid IR (2009) Osteonecrosis of the jaw: who gets it, and why? Bone 44(1):4-10

5. Lesclous P, Abi Najm S, Carrel JP, Baroukh B, Lombardi T, Willi JP, Rizzoli R, Saffar JL, Samson J (2009) Bisphosphonateassociated osteonecrosis of the jaw: a key role of inflammation? Bone 45:843-852

6. Weycker D, Macarios D, Edelsberg J, Oster G (2006) Compliance with drug therapy for postmenopausal osteoporosis. Osteoporos Int 17:1645-1652

7. Robins, J. (1998) Marginal structural models. 1997 Proceedings of the American Statistical Association. Section on Bayesian Statistical Science, pp. 1-10. Reproduced courtesy of the American Statistical Association

8. Robins JM, Rotnitzky A, Zhao LP (1994) Estimation of regression coefficients when some regressors are not always observed. J Am Stat Assoc 89:846-866

9. Hernan MA, Robins JM (2010) Causal inference, Chapman \& Hall/ CRC monographs on statistics \& applied probability, CRC, p. 70, ISBN 1-4200-7616-7

10. Greenland S, Pearl J, Robins JM (1999 Jan) Causal diagrams for epidemiologic research. Epidemiology 10(1):37-48

11. Pearl J (2009) Causality: Models, Reasoning and Inference, 2nd edn. Cambridge University Press, Cambridge

12. Robins JM, Hernan MA, Brumback B (2000) Marginal structural models and causal inference in epidemiology. Epidemiology 11(5):550-560

13. Hernán MA, Brumback BA, Robins JM (2002) Estimating the causal effect of zidovudine on CD4 count with a marginal structural model for repeated measures. Stat Med 21(12):1689-1709

14. Hernán MA, Brumback B, Robins JM (2001) Marginal structural models to estimate the joint causal effect of nonrandomized treatments. JASA 96:440-448

15. Tonti E (2013) Jiménez de Oya N, Galliverti G, Moseman EA, Di Lucia P, Amabile A, Sammicheli S, De Giovanni M, Sironi L, Chevrier N, Sitia G, Gennari L, Guidotti LG, von Andrian UH, Iannacone M. Bisphosphonates target B cells to enhance humoral immune responses. Cell Rep 5(2):323330

16. Macarol V, Fraunfelder FT (1994) Pamidronate disodium and possible ocular adverse drug reactions. Am J Ophthalmol 118:220-224

17. Fraunfelder FT, Fraunfelder FW, Jensvold B (2003) Scleritis and other ocular side effects associated with pamidronate disodium. Am J Ophthalmol 135:219-222

18. Mbekeani JN, Slamovits TL, Schwartz BH et al (1999) Ocular inflammation associated with alendronate therapy. Arch Ophthalmol 117:837-838

19. Moorthy RS, Valluri S, Jampol L (1998) Drug-induced uveitis. Surv Ophthalmol 42:557-570 